\title{
Grands propriétaires face à la gestion publique de la biodiversité au sein du parc naturel régional de Camargue
}

\author{
Christine Demmer \\ Anthropologue, Centre Norbert Elias, 13002 Marseille, France
}

\begin{abstract}
La gestion de la biodiversité relève aujourd'hui de la gestion de la nature ordinaire. Ce retour sur un espace plus remarquable ne doit pas occulter qu'il permet de poser une question d'intérêt plus général, à savoir que pense et que fait le propriétaire privé lorsqu'on l'interpelle sur sa gestion foncière au regard de la préservation de la biodiversité. Cet article permet ainsi de dépasser une ligne de clivage trop simplificatrice entre public et privé, en montrant en quoi la réinterprétation de cet enjeu n'est pas réductible au statut de propriétaire mais engage des formes d'attachement à la terre qui, partielles ou minoritaires, peuvent constituer autant de supports de l'action publique.
\end{abstract}

La Rédaction

\section{Mots-clés :}

grands propriétaires ; parc naturel régional de Camargue ; biodiversité ; conflit privé/public ; patrimoine

\begin{abstract}
Résumé - Cet article s'appuie sur une enquête menée auprès de grands propriétaires fonciers camarguais entre 2006 et 2007. Dépositaires de riches milieux naturels, ces propriétaires constituent l'un des publics cibles des politiques de conservation de cette zone humide méditerranéenne. L'enquête réalisée dans le cadre d'une recherche pluridisciplinaire visait initialement à mesurer l'écart existant entre les représentations et la gestion de la biodiversité par les propriétaires et celles défendues par les acteurs de la conservation sur le territoire du parc naturel régional de Camargue. Elle a permis $\mathrm{d}$ 'identifier trois profils d'usagers $\mathrm{du}$ foncier dont deux d'entre eux, pour des raisons différentes, considèrent leur patrimoine personnel comme ayant des dimensions collectives, ouvrant sur une sensibilité aux questions de conservation. Cette typologie s'inscrit cependant dans un antagonisme plus global entre acteurs privés et acteurs publics qu'expriment des représentations différentes de la nature et qui affecte la gouvernance environnementale locale. Cet article entend explorer ces désaccords.
\end{abstract}

\section{Keywords: \\ large landowners; Camargue Regional Nature Park; biodiversity; private/ public conflict; heritage}

\begin{abstract}
Large landowners and public biodiversity management in the Camargue Regional Park. This paper presents my contribution to an interdisciplinary study of large landowners in the Camargue initiated by the Tour du Valat Research Centre for the conservation of Mediterranean wetlands. As repositories of a rich natural environment these landowners are the main target of conservation policies in this protected area. To find solutions to halt the loss and degradation of natural habitats, my study aimed initially to assess the gap between the representations and management of biodiversity by the landowners and the type of management advocated by the conservation actors in the Camargue Regional Park. This led to identifying a conflict between the private landowners and public actors of nature conservation regarding the control of the Park, with a resulting serious handicap for an efficient integrated management. In analyzing the conflict, there emerged three profiles of landowner relationship with their land: those who consider it as an economic investment, those who consider it as a farming inheritance, and those who view it essentially as a place for leisure. In conclusion the study showed that despite conflicting positions, a discussion is possible, however, between ecologists and conservation managers on the one hand and some land owners on the other, who for different reasons view their heritage as common heritage.
\end{abstract}

Auteur correspondant : christine.demmer@univ-amu.fr 


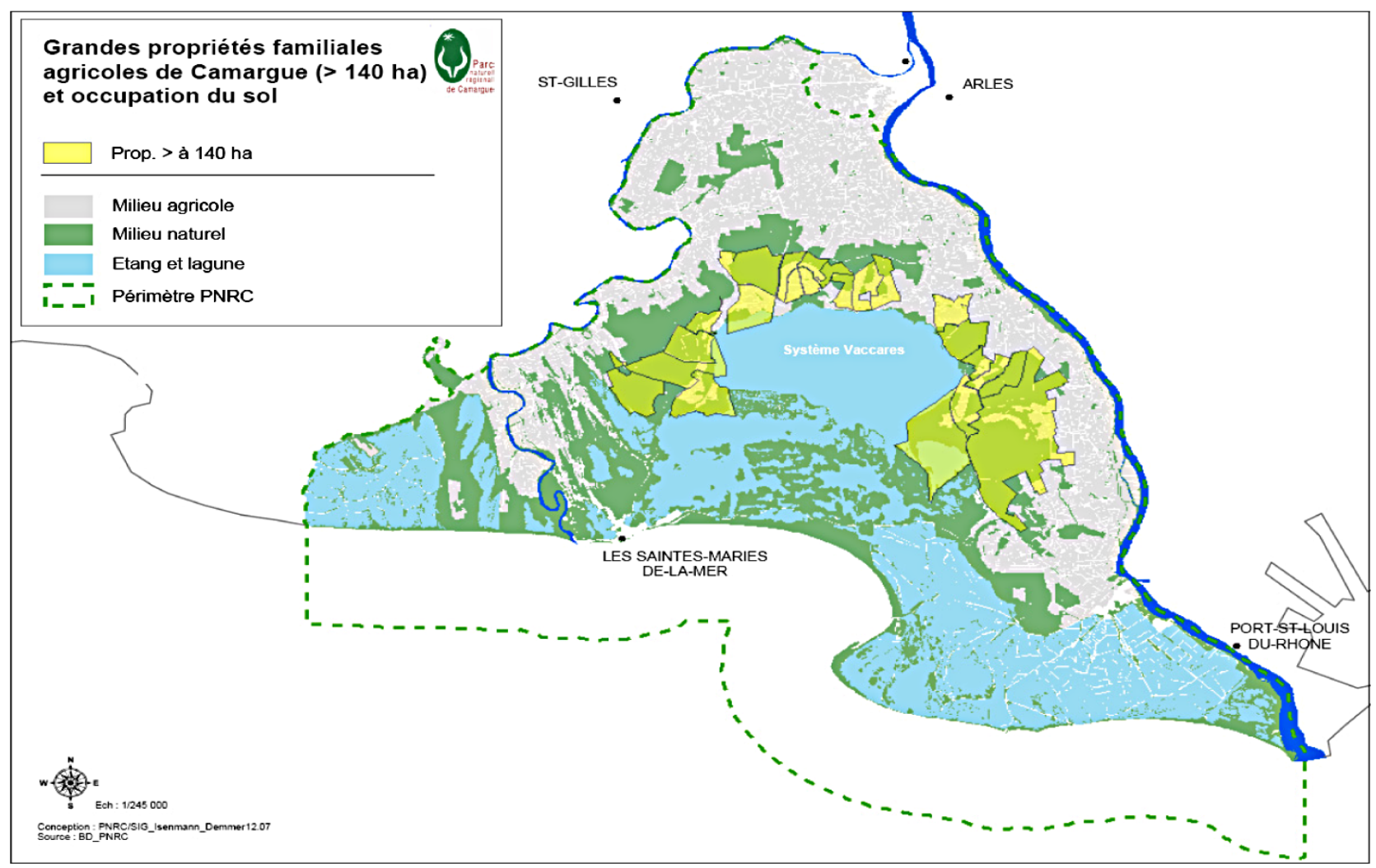

Fig. 1. Très grandes propriétés et milieux naturels.

\section{Penser la collaboration entre grands propriétaires et gestionnaires d'espaces naturels}

Depuis le $\mathrm{XIX}^{\mathrm{e}}$ siècle, tous ceux qui ont côtoyé et/ou aménagé (investisseurs, félibriges ${ }^{1}$, politiciens ou écologues) la Camargue ont contribué à forger la représentation d'un espace socialement et/ou écologiquement menacé (Picon, 1996). Pourtant (ou précisément pour cette raison), ce territoire possède une longue histoire de patrimonialisation de sa nature (Picon, 1978). Ce processus a débuté avec la création de la réserve nationale de Camargue en 1927 (littéralement assimilée à un « monument de nature $»^{2}$ ) et $s^{\prime}$ est prolongé notamment avec le classement en parc naturel régional de Camargue (PNRC) à la fin des années $1960^{3}$. Soucieuse de réduire

1 Le Félibrige est une association littéraire créée en 1854 par Frédéric Mistral et six autres poètes provençaux pour assurer la défense de la culture et de la langue régionale.

2 La réserve fut classée en juin 1942 au titre de la loi du 2 mai 1930 sur la protection des monuments naturels et des sites à caractère artistique, historique, scientifique, légendaire ou pittoresque.

3 Le PNRC fait partie des dix premiers parcs naturels régionaux créés en France. les dégradations environnementales qui se poursuivent malgré cela, la station biologique de la Tour du Valat (TDV), centre de conservation des zones humides méditerranéennes, sis en Camargue, initie des recherches et des projets de conservation. L'une de ces recherches interdisciplinaires concernait les propriétaires fonciers du pourtour du Vaccarès parce qu'ils sont tout à la fois les plus riches dépositaires de milieux naturels (sansouires, montilles et autres roselières qui abritent de nombreuses espèces protégées) et les principaux acteurs de leur transformation par la pratique de la riziculture, de l'élevage, de la chasse, de la sagne, voire par la location de gîtes. Ce sont aussi les propriétaires des espaces fonciers les plus imposants de Camargue avec des propriétés familiales dépassant 140 ha qui recouvrent ainsi 42800 ha de l'espace du parc, contre 5100 ha pour les propriétés de moins de 140 ha $^{4}$ (Fig. 1). Bien qu'interrogés sur leurs connaissances et savoir-faire concernant la

\footnotetext{
4 Vingt-quatre propriétaires du pourtour du Vaccarès sur la trentaine qui possède là une terre ont été interrogés. Les plus gros propriétaires du delta et les plus pourvus en espaces naturels ont été ainsi entendus. Sept gestionnaires et chercheurs se sont aussi prêtés à l'enquête pour donner leur point de vue sur la gestion de la Camargue, celle des propriétaires et leur rapport avec ces derniers.
} 
nature, valorisés depuis la Convention sur la diversité biologique de 1992, les personnes enquêtées préférèrent insister sur le bien-fondé de leurs activités économiques afin de se démarquer de ce qu'ils considéraient comme des injonctions d'écologisation émanant du PNRC et de la TDV. Aussi, une réflexion portant sur les problèmes de gouvernance entre gestionnaires du PNRC et scientifiques, d'un côté, et agriculteurs et éleveurs, de l'autre, s'est alors engagée afin de l'améliorer ${ }^{5}$.

$\mathrm{Au}$ moment des enquêtes, en 2007, une grave crise secouait en effet ce parc naturel régional (PNR). Il était menacé de disparition suite aux divers procès intentés par l'association des propriétaires fonciers de Camargue qui avait perdu le contrôle de l'institution au début des années 2000. Certains grands propriétaires s'opposaient ainsi ouvertement à près de 200 gestionnaires du PNR et chercheurs de la TDV, tous représentants de l'action publique en faveur de l'écologisation du territoire camarguais, dénommés en bloc « écologistes » par les propriétaires privés interrogés ${ }^{6}$. Les propos recueillis auprès des plus grands propriétaires de Camargue (comprenant les plus actifs opposants au parc) mirent en scène ce conflit en critiquant soit la gestion publique du delta, soit sa gouvernance jugée trop autoritaire, tout en faisant état d'une difficulté importante à accepter toute ingérence sur des terres privées. Partant de ce constat, notre recherche s'est orientée vers l'analyse du rapport tendu entre acteurs privés et acteurs publics dans la conservation de la Camargue, là où la sociologue Claeys-Mekdade (2003) questionnait le rapport local/global en s'intéressant aux transformations de l'espace public depuis que les politiques environnementales sont à l'honneur ${ }^{7}$.

\footnotetext{
5 L'interdisciplinarité réside donc ici dans la construction d'un objet de recherche ancré dans l'ethnologie et/ou la sociologie mais dont l'objectif ultime - limiter la perte des habitats naturels - était pertinent pour toutes les disciplines impliquées dans le projet. Voir à ce sujet les définitions de l'interdisciplinarité données par Brun et al. (2007). Je remercie, pour leurs conseils et avis tout au long de cette recherche jusqu'à sa présente finalisation, mes partenaires dans ce projet : Raphael Mathevet (géographe et écologue au CEFE Montpellier), Michel Gauthier Clerc (biologiste à la Tour du Valat), François Mesleard et Arnaud Bechet, (écologues à la TDV). Je me suis appuyée aussi sur les travaux en sciences sociales du laboratoire Desmid (Dynamique écologique et sociale en milieu deltaïque) basé à Arles.

6 La TDV est une fondation reconnue d'intérêt public qui gère 1100 ha de réserve naturelle régionale.

7 Il s'agit de révéler, par l'analyse du conflit, les logiques sociales de l'action. Ce projet peut compléter celui de la recherche des modalités juridiques de conciliation entre intérêt privé et intérêt général appliqué au domaine de la protection de la nature. Je renvoie à Hostiou (1994), Ostrom (1990) ou encore Ostrom et al. (1999) concernant les arrangements institutionnels entre les différents porteurs d'enjeux sur un même
}

Ce texte commence par montrer que les griefs des grands propriétaires exprimés à l'encontre des " écologistes » sont sous-tendus par le sentiment d'avoir perdu face à eux une part de leur ancienne légitimité dans la gestion du delta du Rhône. C'est cette perte de légitimité qui a été identifiée comme une cause des difficultés rencontrées dans la gouvernance locale. Les enquêtés estimaient majoritairement n'être plus maîtres de leurs espaces fonciers alors même que précisément l'article 544 du Code civil semble leur garantir la souveraineté exclusive sur leurs terres ${ }^{8}$. Ils mettaient en cause la multiplication des incitations des gestionnaires du parc - voire des écologues de la TDV - à s'engager plus avant dans des pratiques écologiques après le changement de mode de gouvernance de l'institution du parc (passé d'une fondation où les grands propriétaires étaient dominants à un syndicat mixte) qui coïncidait de surcroît avec la mise en place du dispositif Natura 2000. Dans cet espace où coexistent des zones d'exploitation et de nombreux espaces protégés, et où les agriculteurs sont à présent conviés à participer à l'écologisation du territoire après avoir été appelés, après-guerre, à «nourrir le monde»(Coulomb, 1992), il ressort donc de l'enquête un fort positionnement des personnes interrogées «en tant que propriétaires privés », ce qui les rendait, au pire, hostiles et, au mieux, méfiants à l'égard de l'intervention du public dans la gestion de leur bien. Quelques rares personnes interrogées (deux ou trois) se démarquaient toutefois du collectif «propriétaires camarguais » et étaient plus ouvertes aux propositions émanant notamment du PNRC. Les autres ont fait preuve d'une défiance quasi structurelle le concernant.

Par ailleurs, ces propriétaires s'opposaient, généralement, en tant que "producteurs de ressources marchandes » aux « producteurs de nature ». Sur ce point, ils pouvaient être rejoints par tous ceux qui n'ont pas été interrogés, plus au nord du delta, et qui se consacrent principalement à la riziculture (présente en Camargue sur 18000 ha en tout). Mais si ces derniers défendent sans nul doute, à l'instar de la plupart des agriculteurs, un principe de réalité qui veut qu'on vive de son activité,

espace. Je renvoie à Subra de Bieusse (1979) au sujet de la création de droits concurrents (tels des servitudes d'utilité publique relatives à la conservation du patrimoine naturel). On peut lire aussi Bellivier et Noiville (2009) qui défendent le passage d'une ère de la propriété à un «âge de l'accès » selon le titre de l'ouvrage de Jeremy Rifkin (2005), ce qui suppose de distinguer propriété exclusive et partage contrôlé avec des tiers de biens matériels comme immatériels.

8 Cette perception contraste fortement avec l'entrelacement de fait des droits sur le sol que les chercheurs s'intéressant au foncier, surtout depuis l'essor des politiques publiques environnementales, ne manquent pas de rappeler. Lire par exemple les travaux de $\mathrm{H}$. Jacobs aux États-Unis assimilant le foncier à un « faisceau de droits » différenciés. 
parmi les grands propriétaires enquêtés, on notait souvent une identification à la figure du « propriétaire travailleur » forgée par John Locke dans son Deuxième Traité du gouvernement civil de 1689. En l'occurrence, ils affirmaient le droit fondamental d'exploiter leur terre comme bon leur semble pour assurer leur survie, garantissant du même coup leur liberté individuelle. Car dans l'optique de Locke, seul celui qui transforme la nature est un homme libre. En se positionnant ainsi, ces propriétaires entendaient qu'on respecte leurs choix. Ils défendaient le productivisme non pas tant dans une simple logique financière, mais bel et bien au nom d'une idéologie libérale liant propriété et travail. À cet égard, même si les intéressés aimaient à souligner qu'un propriétaire prend soin de ce qui lui appartient bien plus que celui qui n'en possède que l'usage, l'image du propriétaire privé "responsable", soucieux de son milieu quasi par essence, défendue par les "écologistes de marché » ne tient pas ${ }^{9}$. L'idéologie libérale soutenue dans les termes évoqués les conduisait plutôt à un usage intensif du foncier. Le souci de rentabilité affiché par bon nombre d'exploitants, identifié par les écologues qui travaillent dans cette zone humide méditerranéenne protégée comme étant le principal problème à résoudre (Mathevet et al., 2002), prend donc ici un relief particulier avec la défense des idéaux de la Révolution française.

Mais si ce profil du propriétaire productiviste s'impose peut-être ici avec plus de force qu'ailleurs, du fait de l'existence d'une catégorie relativement homogène - celle de grands propriétaires camarguais défenseurs du privé contre le public qui peuvent aussi défendre ardemment la valeur travail -, cette recherche fait aussi état d'usages du foncier qui se démarquent du productivisme. On trouvait en effet des personnes qui revendiquaient leur statut de propriétaires et se méfiaient des "écologistes", mais qui s'avéraient soucieuses, en pratique, d'une gestion moins prédatrice de leurs terres. Dans le prolongement de François Ost (2003) - qui voit dans le patrimoine un concept riche de potentialités pour créer " un statut juridique pour le milieu », permettant de faire converger intérêt privé et politique publique quand celle-ci entend, comme en Camargue, «écologiser » plutôt que "moderniser »-, il a été cherché ce qui permet, chez les propriétaires, d'entrevoir le bien foncier comme patrimoine commun, ouvrant sur une « responsabilité » environnementale. La piste suivie consista à déterminer la place qu'occupe le foncier dans le patrimoine général des propriétaires ainsi qu'à

9 La nouvelle économie des ressources (new resource economics) dite encore écologie de marché (free market environmentalism) est un courant d'inspiration très libérale qui prône la privatisation des ressources afin de mieux les conserver. L'idéologie de la conservation privative s'appuie sur la thèse de Hardin (1968). Voir Terry et Leal (2001) ou Bromley (1991). interroger l'histoire du foncier dans les trajectoires personnelles et familiales. C'est ainsi que trois profils de propriétaires ont été identifiés qui conditionnent trois types d'usages de la terre.

\section{Des grands propriétaires influents en perte de légitimité}

En Camargue, la grande propriété est relativement pérenne depuis le XVIII ${ }^{\mathrm{e}}$ siècle. Parce que l'aménagement de ces terres salées et inondables est coûteux, elles restent de taille remarquable. Généralement comprises entre 300 ha et 500 ha, certaines de ces propriétés atteignent même 3000 ha aux abords de la réserve et du Vaccarès. Aujourd'hui, les propriétés privées recouvrent plus de $70 \%$ des 85690 ha du PNRC de l'époque, le reste de la superficie relevant d'espaces naturels gérés par le public (Fig. 2). Forts de cette emprise foncière, les propriétaires jouent un rôle central dans la dynamique socioéconomique du delta. Les treize plus grosses exploitations agricoles (dont certaines se situent autour du Vaccarès) recouvrent près de $30 \%$ du territoire du parc. L'un des propriétaires rappelait : « On fait 900 millions de chiffre d'affaires en euros ${ }^{10}$.»

Ces grands propriétaires appartiennent aux catégories sociales aisées ; certains fréquentent les lieux de pouvoir. Cette configuration sociale est ancienne. Après le clergé - présent depuis le $\mathrm{XI}^{\mathrm{e}}$ siècle - sont venus des aristocrates puis, au XVIII ${ }^{\mathrm{e}}$ siècle, une centaine de propriétaires forains (bourgeois riches commerçants et/ou industriels des environs d'Arles, de Lyon ou Marseille (Allard, 1978). Ces familles ont été rejointes au XXe siècle par des descendants de fortunes coloniales et enfin par des fortunes immobilières ou financières. Souvent assujettis à l'impôt sur les grandes fortunes, ces propriétaires disposent d'un patrimoine global dont le foncier camarguais peut représenter une part non négligeable mais qui est concurrencé, chez certains, par d'autres biens immobiliers et mobiliers importants.

Rien d'étonnant dans ce contexte qu'à l'ère de la modernisation de la France, l'île de Camargue - où Pompidou chassait en compagnie de certains propriétaires - fut pressentie comme espace dévolu à la contemplation de la nature. Espace compris entre Fos-sur-Mer et La Grande-Motte, il se devait de préserver la tranquillité de ces grands propriétaires tout en n'entravant pas trop leurs activités économiques. Pour ce faire, la gestion du parc leur fut confiée via une fondation ${ }^{11}$. La

\footnotetext{
${ }^{10}$ Mais $40 \%$ des revenus des Camarguais dans leur ensemble proviennent des aides de l'État et de l'Union européenne (Mathevet et al., 2002).

${ }^{11}$ Huit des membres de l'association appelée «Comité des propriétaires camarguais » siégeaient au conseil d'administration avec les collectivités locales et les services publics (PNRC, textes constitutifs, 1973).
} 


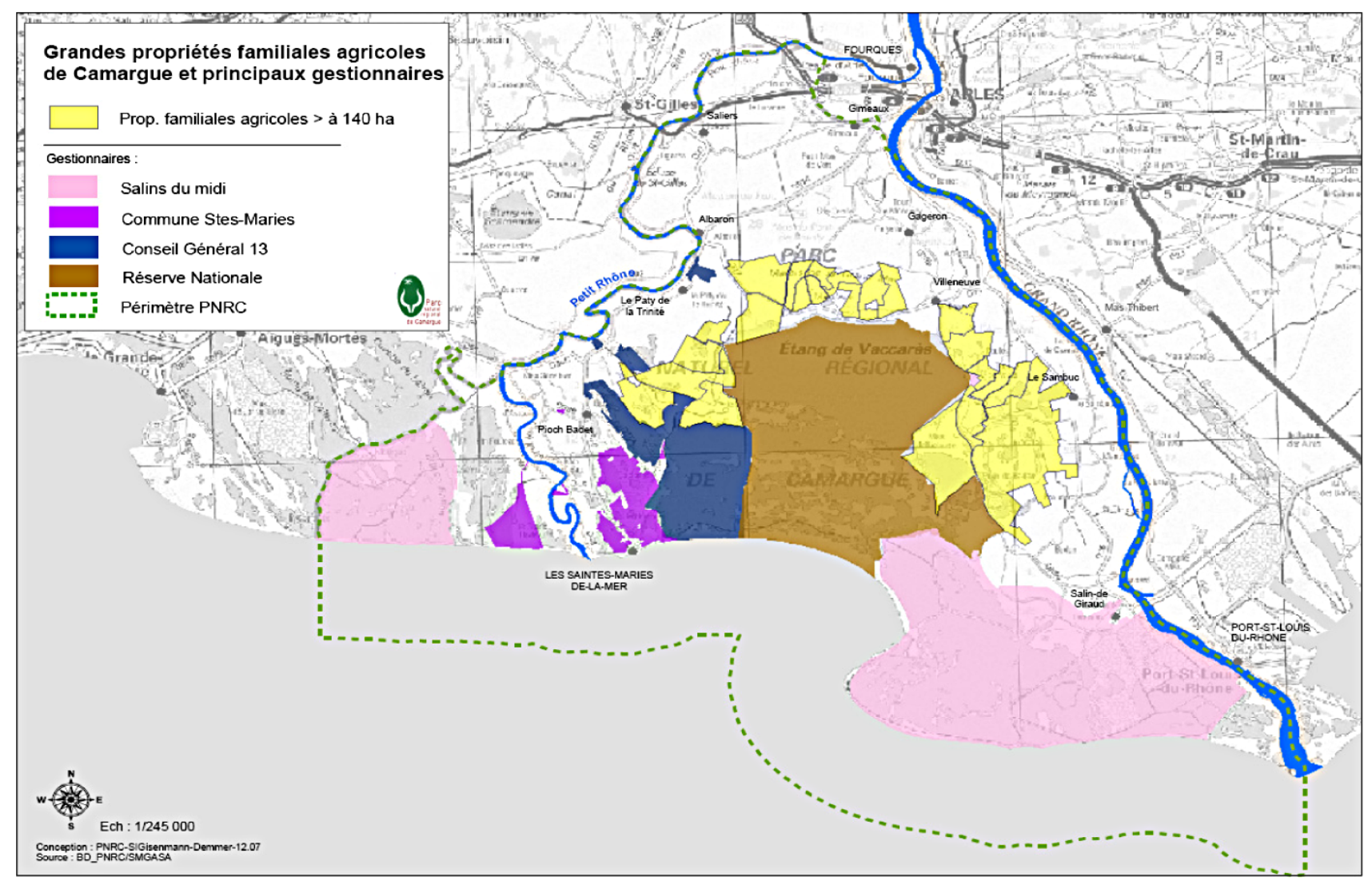

Fig. 2. Gestionnaires publics d'espaces naturels.

dissolution de cette dernière en 2001 lors de la mise en place d'un groupement d'intérêt public (avant la création du syndicat mixte en 2004, le Conseil d'État estimant cette dernière structure seule apte à gérer un parc naturel régional) est donc venue contrecarrer leur suprématie sur ce territoire. Il faut garder à l'esprit qu'au temps où prévalait encore un mode de production paternaliste, les ouvriers agricoles vivaient sur la propriété ; les propriétaires étaient considérés comme des « seigneurs » de la Camargue. L'adoption du texte relatif au syndicat mixte de gestion du parc naturel régional à l'unanimité à l'Assemblée nationale en 2007 leur a toutefois redonné une place dans le jeu politique local ${ }^{12}$. Mais, au moment des enquêtes, ce règlement du problème juridique, alors en cours, n'était pas encore intervenu. On repérait nettement dans les critiques formulées à l'endroit de la gestion du PNRC une forme d'amertume liée à une maîtrise perdue de l'espace. Cet état de fait tendait à rejeter les

\footnotetext{
${ }^{12}$ Le texte propose aux propriétaires (de même qu'aux représentants du monde agricole) un mode de participation aux instances délibératives au sein du comité syndical du parc, et non pas seulement consultatives, sous la forme de la participation de la fondation via une association syndicale autorisée (Asa).
}

" écologistes », personnes sans assise foncière, détentrices d'un savoir livresque, traités de " Parisiens bardés de diplômes » qui ne compenseront jamais la connaissance empirique.

Pour les grands propriétaires camarguais interrogés, le capital culturel ne pesait guère. La légitimité à orienter l'aménagement du delta (et donc à garder la maîtrise sur le PNRC) était affaire de possession de terres associée à un capital économique, voire fonction d'une grande surface sociale. La référence à Locke servait à exprimer, à l'instar du philosophe du XVII ${ }^{\mathrm{e}}$ siècle, l'idée que pour pouvoir exister comme un individu indépendant vis-àvis du corps social et politique, il faut être propriétaire (Castel et Haroche, 2001, p. 35). La grande propriété a ainsi souvent été affichée par les enquêtés comme la justification principale du droit à s'emparer des affaires de la Cité, à l'image de la période qui suivit la Restauration où la citoyenneté ne revenait quasiment qu'aux seuls propriétaires (Castel et Haroche, 2001, p. 39). La création d'un syndicat mixte apparaissait à cet égard une hérésie, laissant entrer dans la gestion du parc de Camargue les «simples» locataires ou les très petits propriétaires ou encore les salariés des mas :

« Le parc régional n'existe pas. Il n'a pas de sol. [...] On a fait entrer la population des hameaux dans le conseil 
d'administration. Juste des habitants qui ont des petites maisons, des choses comme ça... qui ont une voix aussi importante qu'un propriétaire qui a 500 ha. On a noyé le système décisionnaire. Les gens vraiment propriétaires du sol qui doivent payer des charges et gérer ont moins leur mot à dire. »

Se jouait ici une forme de conflit de classes entre, d'un côté, des propriétaires fonciers qui possèdent leurs moyens de production et, de l'autre, des résidents qui ne les possèdent pas. Ces derniers étaient significativement dénommés «habitants », pour bien montrer leur absence d'assise foncière solide. Envers les "écologistes ", la démarcation rendait palpable une certaine animosité pour cause de ravissement du pouvoir sur le delta. Logiquement, ceux qui ne se reconnaissaient pas dans la catégorie de «propriétaires» qualifiaient cette vision «d'élitiste ». Pour paraphraser Rousseau, ils considéraient que si l'homme est né libre, il est aussi dans sa nature d'être social et de partager le pouvoir ${ }^{13}$. Quant aux gestionnaires du PNRC, ils reprenaient ces catégories à leur compte, comme s'il s'agissait de dénominations allant de soi : comme si le grand propriétaire n'était pas aussi un habitant et l'habitant, éventuellement, aussi un propriétaire.

L'existence du Comité des propriétaires camarguais ${ }^{14}$ qui géra la fondation du PNRC a joué un rôle incontestable dans la construction de cette catégorie de « propriétaires » qui comprend tout particulièrement les plus dotés en espaces fonciers (précisément ceux interrogés sur le pourtour du Vaccarès). Mais en trente ans, cette association a eu tout loisir d'imprimer sa vision de l'aménagement du territoire bien au-delà du cercle de ces personnes. L'éviction du comité des propriétaires de l'institution PNRC en 2001 a manifestement renforcé la cohésion d'un groupe sinon plus lâche. D'ailleurs, ceux qui, au moment des enquêtes se revendiquaient légitimes à gérer le parc n'étaient pas ceux qui, historiquement, contribuèrent à le faire exister (tous issus toutefois de la même zone laguno-marine). Au fil du temps, le discours consistant à défendre la grande propriété au nom de la protection de la nature s'est couplé - du fait de nouveaux acteurs animant la fondation - d'un message de défense du productivisme qui n'était pas le cheval de bataille du noyau dur des fondateurs du parc ${ }^{15}$. Ce

\footnotetext{
${ }^{13} \mathrm{Ce}$ postulat rousseauiste fut opposé du temps de la Révolution à celui de Locke (Capitan, 2000, p. 71).

14 Cette association acceptait (en principe plus qu'en pratique) toute personne physique ou morale possédant au minimum 5 ha.

15 Lors des discussions en commission du parc mais aussi via le Centre français $\mathrm{du}$ riz, les associations syndicales autorisées de drainage ou d'arrosage, ou encore lors des réunions du syndicat des riziculteurs ou celles de la chambre d'agriculture.
}

message, susceptible de rencontrer un écho favorable au nord, dénotait autant une forme d'amertume résultant de la perte de pouvoir, qu'il exprimait des divergences d'option pour la gestion du delta avec les acteurs publics du PNRC.

La frange la plus libérale de l'échantillon était à cet égard rejointe par d'autres, plus anciennement implantés, pour juger les "écologistes » comme étant des socialistes, étatistes, partisans d'une société utopique (parce qu'anti-productiviste). La dissolution de la fondation du PNRC était perçue comme «un coup politique » initié par Michel Vauzelle, président PS de la région Paca et député des Bouches-du-Rhône, désireux de faire entendre la voix des «simples habitants » et de mener une "croisade pour l'étatisation de la Camargue ». L'action publique en faveur de la protection de la nature se résumait pour les plus virulents à une stratégie délibérée d'éviction de la grande propriété et/ou de la libre entreprise afin d'instaurer un «système communiste déclarant la guerre à la grande propriété ». Pour les plus modérés, il s'agissait plus prosaïquement de réclamer plus de respect de la part des «écologistes » et de défendre la « sociodiversité » contre ce qui apparaissait comme une défense excessive de la biodiversité. En somme, il s'agissait de faire accepter le maintien des activités lucratives, sans en être pour autant déconsidéré.

Une plus grande considération était la condition de l'établissement d'un dialogue apaisé concernant la mise en œuvre de pratiques plus écologiques. En l'état des relations, le sentiment de déclassement conduisait la majorité à renforcer la référence à la liberté $\mathrm{d}^{\prime}$ action $\mathrm{du}$ propriétaire : «La propriété privée, c'est la propriété privée. Je suis chez moi et je fais ce que je veux ", pouvait-on entendre. La mise en place du dispositif Natura 2000 dans le delta du Rhône, pour lequel le PNR de Camargue est gestionnaire des sites, cristallisa ce genre de réactions épidermiques. Les rares personnes qui ne se reconnaissaient pas dans le statut de propriétaire, étaient bien entendu plus enclines à accepter l'idée de protéger les habitats naturels situés sur leurs terres. Mais pour les autres, Natura 2000 - qui semble avoir multiplié les contraintes de gestion - était perçu comme portant atteinte à l'intégrité de la propriété et à la souveraineté du propriétaire sur ses terres au point de susciter l'envie de "mettre le feu aux roselières » ou encore de "défricher les enganes ». L'interventionnisme sur le foncier privé était critiqué à hauteur des aides financières obtenues personnellement ou via le parc, grâce à une Politique agricole commune qui, selon les propos même d'un enquêté « continue à subventionner les gros céréaliers du centre de la France et les gros riziculteurs camarguais ». Tout se passait comme si, plus la dépendance financière était forte, plus les grands propriétaires se 
devaient de réaffirmer leur droit à la « liberté », intrinsèquement lié à leur statut de propriétaire ${ }^{16}$.

\section{Des représentations de la nature rejouant l'antagonisme privé/public}

L'opposition privé/public, revenant à revendiquer à la fois une meilleure place dans la gouvernance locale et un plus grand respect de la souveraineté foncière, prenait aussi la forme d'un désaccord relatif aux choix d'aménagement du territoire et des propriétés d'un PNR par ailleurs en zone Ramsar, réserve de biosphère et site Natura 2000. Pour l'ensemble de l'échantillon, il était important d'affirmer la nécessité de gagner décemment sa vie, en demandant à inscrire dans les orientations de gestion du territoire du parc le couplage des activités économiques à la protection de l'environnement. De manière parfois simpliste, ceux qui se revendiquaient comme «travailleurs » s'opposaient aux « contemplatifs ». C'était d'autant plus le cas dans la tranche d'âge la plus élevée de l'échantillon ${ }^{17}$ et/ou la plus libérale : les premiers étaient imprégnés du discours de modernisation de l'agriculture; les seconds, toujours dans la logique d'adhésion aux idéaux de la Révolution française liant travail et propriété, défendaient la productivité. La campagne présidentielle de 2006 qui battait alors son plein, porteuse du slogan à succès "travailler plus pour gagner plus ", n'était sans doute pas étrangère à l'affirmation forte d'un discours productiviste au moment des enquêtes ${ }^{18}$.

Mais pour défendre leurs activités et leur place au sein du parc, les propriétaires faisaient état de représentations de la nature différentes de celles des «écologistes ${ }^{19}$. Ils valorisaient l'anthropisation - mais raisonnée - supposée conférer leur beauté aux paysages

\footnotetext{
${ }^{16}$ La posture des propriétaires - qui consiste à revendiquer haut et fort les subsides publics tout en les dénigrant - est ancienne ici. On la constate par exemple dès le Second Empire avec la construction de la digue à la mer qui s'inscrit dans l'optique de l'amélioration des performances agricoles en Camargue (Pailhès, 2000).

17 Cela concerne donc la plupart des enquêtés puisque la moyenne d'âge de l'échantillon dépassait 56 ans en 2007. La moitié exactement avait plus de 60 ans ; sur cet effectif restreint, la moyenne d'âge atteignait près de 70 ans.

18 Nicolas Sarkozy ne s'est pas rendu pour rien en Camargue juste avant son élection ; il avait ici de fidèles supporters. À noter que la valeur travail est sans doute renforcée ici par l'existence $\mathrm{d}^{\prime}$ une forte communauté protestante, religion dont on sait depuis Max Weber le rôle qu'elle a joué dans l'essor du capitalisme.

${ }_{19}$ Cela fait écho à ce que les sociologues Kalaora et Savoye (Cadoret, 1985) constatent dans les controverses environnementales: "Les représentations de la nature de différents groupes sociaux [...] expriment des conceptions et des stratégies d'appropriation spatiale conflictuelles. »
}

camarguais. Les propriétaires estimaient en premier lieu que les écologues et gestionnaires du PNRC exagéraient le problème de l'artificialisation du milieu, considéré comme nuisible aux habitats spécifiques méditerranéens. Ils défendaient leur mode de gestion hydraulique face à des "écologistes " visant un moindre interventionnisme, préconisant le respect d'une alternance sec/ humide, propre au fonctionnement naturel de cette zone humide. À l'inverse, la plupart des riziculteurs et des chasseurs valorisaient une maîtrise hydraulique constante, marquée par un apport régulier en eau douce. Les premiers défendaient cette option destinée à lutter contre la salinisation du sol en rappelant qu'elle évitait «la désertification de la Camargue ». Les seconds vantaient la production de biomasse (des herbiers attirant une avifaune gibier abondante). Quant aux éleveurs, ils se souciaient surtout de perpétuer des lignées taurines et équines locales. La notion de biodiversité était ici réduite à l'abondance de certaines espèces, renvoyant les écologistes à leurs débats internes et non résolus concernant les espèces indicateurs de biodiversité : "Quoi protéger et pourquoi protéger? Il faut qu'on nous explique!» Pour tous les propriétaires, il était important de privilégier les éléments de la nature susceptibles de garantir un revenu. Variétés de riz, herbiers à canards, pelouses, sansouires et roselières trouvaient grâce à leurs yeux en tant que «ressources utiles». Cela n'excluait pas chez certains une attention particulière aux milieux spécifiques à la Camargue, forme de diversité biologique valorisée parce qu'identitaire. Mais les propriétaires survalorisaient surtout massivement ce qu'on peut nommer - en miroir du terme de biodiversité -, une certaine sociodiversité. Ils appréciaient la coexistence dans l'enceinte du parc d'activités agricoles, pastorales et cynégétiques, jugées harmonieusement réparties, précisément grâce à une gestion hydraulique active reposant sur une grande variabilité de hauteur et de durée de mise en eau. Par la diversité des activités exploitantes, les propriétaires se targuaient de produire des paysages et des espaces divers, bien qu'anthropisés. Les pluriactifs soulignaient ainsi leur capacité à maintenir une mosaïque de milieux sur leurs propres terres. Les arguments mobilisés par les propriétaires pour défendre leur gestion se situaient ainsi sur le thème - rendu incontournable par les « écologistes »-de la diversité, mais employés plus pour défendre des usages fonciers qu'une diversité biologique habilement ramenée du côté d'une diversité des paysages. La défense de cette dernière avait de surcroît l'avantage de valoriser une synthèse de l'identité camarguaise, renvoyant aussi bien à son versant naturel (ses habitats spécifiques) que culturel (symbolisé par ses activités pastorales et équines).

Les grands propriétaires estimaient que les zones protégées existantes suffisaient bien à maintenir les habitats représentatifs de la Camargue et/ou des zones 
humides ${ }^{20}$. À l'exception des manadiers qui peuvent exercer leurs activités sur les terres publicisées, les propriétaires se plaignaient de leur extension. Convaincus d'être des protecteurs de l'environnement, ils mettaient en doute l'érosion de la biodiversité ou la régression des habitats naturels constatées par les "écologistes " (Tamisier, 1990 ; Tamisier et Grillas, 1994). Seuls les salins méritaient ici le label « artificiels » parce que fruits d'une transformation industrielle. Certains estimaient même être "producteurs de milieu naturel ». Les riziculteurs - les plus stigmatisés - se faisaient les chantres d'une production dite "raisonnée ", à la fois rationnelle (fondée sur la technologie agricole et chimique au service $\mathrm{du}$ rendement) et raisonnable (utilisant moins d'engrais et de pesticides que dans les années 1950 jusqu'aux années 1960 et 1970, et respectant les terres basses). Ils se gardaient bien de souligner le fait que le plan de relance de la riziculture fut porté par la fondation du PNRC - donc par les grands propriétaires - au début des années 1980. Les personnes interrogées préféraient rappeler que deux tiers d'entre elles avaient souscrit aux mesures agri-environnementales en précisant que cela n'avait pas changé leurs habitudes de travail ${ }^{21}$.

En réalité, pour tous les propriétaires, la vraie menace environnementale en Camargue était celle du morcellement du foncier privé (peur qui rejoint celle de l'appropriation publique du foncier privé) ${ }^{22}$. Ils estimaient que depuis la création du syndicat mixte du PNRC en 2004, remplaçant la fondation reconnue d'utilité publique aux mains des propriétaires, le parc encourageait la densification des hameaux et se positionnait pour la transformation de Salin-de-Giraud en station balnéaire. Le conseil régional et la ville d'Arles (de gauche) étaient accusés de cautionner un tourisme de masse. La confrontation de ces critiques aux orientations défendues tout au long des trente années de contrôle de l'institution PNR par le Comité des grands propriétaires démontrait une grande constance dans la lutte contre ces options d'aménagement, qui se couplait à une politique d'acquisition foncière privative autour du Vaccarès, lieu stratégique de la maîtrise hydraulique ${ }^{23}$. La fondation s'était aussi

\footnotetext{
20 La réserve nationale de Camargue créée dans les années 1920 compte 13000 ha. Depuis une vingtaine d'années, d'autres acteurs publics acquièrent des terrains : les conseils généraux du Gard et des Bouches-du-Rhône ainsi que le Conservatoire du littoral. La Fondation Tour du Valat achète elle aussi, régulièrement, de grandes superficies en vue de la conservation.

21 La plupart des propriétaires ont tout de même abandonné la gestion MAE/CTE lorsque les subventions se sont taries.

22 La densité moyenne au sein du parc est de 10 habitants au $\mathrm{km}^{2}$. C'est la plus faible de la région Paca.

23 Voir les comptes-rendus des conseils d'administration de la fondation du parc, consultés au centre de documentation du PNRC.
}

systématiquement opposée aux propositions de zonage du territoire du parc qui desservaient les propriétaires. Ces derniers avaient effectué des demandes répétées d'exonération d'impôts sur le non-bâti ; ils tentèrent encore de mettre en place « l'article 19 » (la prime écologique pour comportement « respectueux » de la nature). Ce faisant, ils ont globalement œuvré dans le sens du maintien et de la valorisation de la grande propriété au nom de la défense de la nature - préservant dans le même temps un « entre soi » social.

Sur ce point, ils étaient rejoints par les «écologistes » qui adhéraient à l'idée que la grande propriété protège l'environnement contre l'urbanisation forcenée. En revanche, l'argument concernant le rôle actif des propriétaires dans la diversité paysagère camarguaise ne convainquait pas ces derniers ; il servait pour eux de caution à une exploitation par trop intensive, sous couvert de défense d'une certaine diversité biologique. En réalité, eu égard à la question du productivisme, trois profils de propriétaires se sont dégagés nettement, allant $\mathrm{d}$ 'une vision individualiste à une vision patrimoniale du foncier, plus favorable au dialogue avec les instances du parc et la TDV.

\section{Trois profils d'usages}

Les propriétaires qui n'étaient qu'accessoirement cultivateurs et qui exerçaient (ou avaient exercé) un ou plusieurs autres métiers comptaient parmi les plus virulents opposants aux « écologistes ». Principalement riziculteurs, ils pratiquaient aussi la location de chasse et n'avaient qu'un faible enracinement en Camargue (une à deux générations). Certains étaient des bourgeois du commerce et de l'industrie ayant acheté après-guerre, d'autres étaient des descendants de colons venus en Camargue au moment des décolonisations (ou un peu avant). D'autres encore avaient réussi plus récemment dans l'immobilier ou dans la finance. Certains d'entre eux résidaient toutefois en Camargue (essentiellement les descendants de colons du Maghreb ou d'Afrique noire). Cette catégorie d'investisseurs dessinait un profil $\mathrm{d}^{\prime}$ « entrepreneurs ». L'un des membres de cette catégorie, vivant à Marseille, fortune du commerce portuaire, se définissait explicitement comme un "financier » et non comme un "agriculteur». Lui, comme les autres, prenait appui sur des régisseurs, «vrais professionnels » aptes à jongler avec le sel et les crues, opposés aux « amateurs ». Ils se disaient « gestionnaires de la nature », pour faire écho aux "plans de gestion » mis en œuvre dans l'écologie contemporaine. Ils défendaient avec force l'idéal productiviste. Cela les conduisait dans le même temps à dénigrer les savoirs locaux, bons pour les agricultures de subsistance: "Il ne faut pas que l'on devienne des sortes $\mathrm{d}^{\prime}$ Indiens. Il se trouve que mes amis 
ne sont ni éthyliques, ni sidaïques, ni syphilitiques. Ils sont en pleine forme, entrepreneurs et fiers de l'être. » L'image de la nature «mère providentielle » cédait chez eux la place à la nature artificielle, transformée par le recours massif aux intrants (pesticides et engrais) et à la mécanisation, apparentant leur agriculture à une forme d'exploitation industrielle. Membres - voire dirigeants de la chambre d'agriculture ou encore du Centre français du riz, ces propriétaires, même s'ils n'étaient pas les plus nombreux (ils représentaient $26 \%$ de l'échantillon, en parts égales avec le profil de "rentiers », soit sept personnes), étaient alors très influents. Ce sont eux qui tendaient à renvoyer dos à dos « grands propriétaires » et « écologistes » en faisant valoir leur légitimité de propriétaires, aptes à faire les bons choix en matière d'aménagement du delta.

La propriété camarguaise était pour eux une ressource - un capital - parmi d'autres qu'il s'agissait de rentabiliser au même titre que leurs actifs mobiliers ou leurs biens immobiliers. Ces propriétaires possédaient peu de connaissances sur leur milieu. Ils n'avaient pas d'attache sentimentale aux lieux: "Je peux vendre demain sans problème. Pour moi c'est du «business », expliquait un descendant de pied-noir. Le rapport à la nature des membres de cette catégorie d'individus s'avérait distancié. Le patrimoine foncier apparaissait avant tout comme un bien qu'on transmet en tant que tel, sans transmettre une identité (un métier et des savoir-faire ou encore l'image d'un lieu qui peut faire rêver pour sa nature et son folklore). Il n'y avait pas là place pour une conception de la propriété sous la forme d'un patrimoine commun. Peu sensibles aux politiques publiques environnementales, ils se faisaient les chantres de la libre entreprise.

Les exploitants se définissant comme « agriculteurs » tiraient l'intégralité de leurs profits du foncier camarguais. Ils étaient soit pluriactifs (riziculture, élevage, sagne, location de gîtes), soit plus rarement grands éleveurs se consacrant surtout à sélectionner des taureaux pour les arènes et autres courses taurines (certains sont aussi tournés vers le tourisme). Leur diversification était le fruit de l'abondance de terres basses (avec sansouires, marais et roselières) sur leur propriété. Bien représenté sur le pourtour du Vaccarès, ce type de terres a eu pour effet de faire gonfler cet effectif dans l'échantillon (ils étaient près de $47 \%$ soit dix personnes interrogées), alors même que sur l'ensemble du delta la première catégorie est bien mieux représentée. Ces propriétaires étaient moins fortunés que les précédents ; leur origine sociale était plus modeste. Le foncier camarguais représentait la part principale de leur patrimoine global. Bien familial, soit possédé depuis plus de quatre générations, soit acquis par les ascendants directs, sa superficie n'était pas forcément inférieure à celle de la catégorie précédente. Issus de familles d'agriculteurs ou de gardians, voire de petits commerçants provençaux, ils faisaient figure, avant la Seconde Guerre mondiale de véritables chefs d'entreprise régnant sur un personnel fixe ou temporaire plus qu'ils n'étaient hommes de terrain (Audema, 1975).

Tous revendiquaient explicitement un savoir-faire agricole et tenaient à se démarquer de la posture technicoscientifique des «entrepreneurs». Lorsqu'ils étaient attirés par les labels de qualité, c'était dans une démarche plus écologique qu'économique. Tout en disant vouloir s'inscrire ainsi dans une continuité culturale ancestrale, ils rompaient toutefois, de fait, avec les logiques de production intensive celles de l'industrie agroalimentaire auxquelles avaient adhéré leurs parents. Mais cela leur était coûteux dans la mesure où la pluriactivité (jugée "mieux adaptée à la Camargue») est moins subventionnée que la riziculture. Cela les obligeait parfois à « faire en fonction des primes ». Pour eux, la terre était perçue comme un bien particulier, privé, mais aussi spécifique à ce milieu humide méditerranéen. Le foncier s'inscrivait donc dans une double conception patrimoniale: familiale et naturelle. Il fonctionnait comme marqueur d'identité sociale et culturelle. Il conférait à son possesseur son statut d'agriculteur et de Provençal et/ou de Camarguais. Les éleveurs (manadiers et gardians) s'identifiaient au mythe de la bouvine qu'ils incarnent et transmettent. Les pluriactifs, agacés par cette image, faisaient valoir l'antériorité de leurs pratiques (élevage de moutons et vignes) ${ }^{24}$. Ils s'emparaient plus volontiers du folklore autour des costumes arlésiens. Mais agriculteurs et éleveurs se retrouvaient pour défendre le versant écologique du mythe camarguais, celui qui valorise les habitats spécifiques et véhicule l'image du «paradis pour les oiseaux ». Or, cette valeur identitaire, ajoutée à la valeur marchande de la terre, favorise un usage plus modéré du sol et une plus grande attention pour le maintien des "terres incultes». Cela conduit à tenir des propos comme : "On veut garder la propriété comme on l'a toujours connue » ou «Il faut laisser les choses comme elles sont». Mais leur gestion " équilibrée » (entre valorisation des milieux cultivés et préservation des milieux "sauvages ») doit être aussi mise en lien plus prosaïquement avec les importants coûts d'aménagement des terres basses. Quoi qu'il en soit, les « agriculteurs » apparaissaient plus ouverts aux propositions des protecteurs de la nature. Mais une collaboration n'était envisageable qu'à la condition que «les spécialistes apportent leur aide aux gens du pays et pas le contraire ». La gestion du PNRC par le syndicat mixte actuel n'était donc pas honnie sur le fond (pour ses propositions) mais elle se devait de respecter

\footnotetext{
${ }^{24}$ Les premières manades qui s'installent en Basse Camargue datent de 1855-1860, à peu près au même moment où s'installe la Compagnie des produits chimiques d'Alais et de la Camargue qui deviendra la $\mathrm{C}^{\text {ie }}$ Pechiney.
} 
les propriétaires en les impliquant mieux dans la gouvernance locale.

Les grands bourgeois issus de fortunes de l'industrie et du commerce de la fin du XIX ${ }^{\mathrm{e}}$ siècle et de la première partie du $X X^{\mathrm{e}}$ siècle, sociologiquement plus proches $\mathrm{du}$ profil des «entrepreneurs » que de celui des "agriculteurs », peuvent être rapprochés de ces derniers par leur conception patrimoniale de la terre. Parmi eux figurent les fondateurs du parc. Il est possible de les qualifier de " rentiers », indépendamment de leur situation réelle, mais relativement au niveau de fortune initial de l'acheteur. Le foncier camarguais fut un placement - une réserve financière. Il fonctionna aussi comme lieu d'agrément. Les activités développées sur la propriété de nos jours semblent viser moins la rentabilité que la neutralisation des coûts engendrés par la possession ancienne d'une telle surface. Un héritier rappelle que son ancêtre s'était offert une propriété immense " où il venait chasser deux fois par an " à une période «où la Camargue n'intéressait personne ${ }^{25}$. Ces riches propriétaires - ne vivant pas dans le delta - sont encore souvent liés aux plus grands éleveurs qui leur offrent du plaisir en échange de pâturages conséquents ${ }^{26}$. Un acheteur récent qui se percevait comme un mécène pour les manadiers expliquait : "L'élevage, c'est une danseuse magnifique qui fait rêver. » L'enracinement des plus représentatives de ces personnes dans le delta correspond significativement aux moments clés où s'est forgé et renforcé le mythe camarguais du manadier ou du gardian, bohème ou poète réalisant une passion. Leur achat date du début $\mathrm{du} X X^{\mathrm{e}}$ siècle, à l'époque de Mistral et de Baroncelli, ou d'après-guerre, du temps de l'écrivain et scénariste Denys Colomb, coauteur du film Crin blanc. Nulle position de principe contre l'entrepreneuriat ne les animait ; leurs origines sociales les ancraient tout à fait dans ce monde. Ils faisaient simplement valoir une moindre nécessité de rentabilisation, liée quelquefois à une moindre possibilité d'exploiter le sol (pour cause d'abondance de terres basses sur leurs propriétés).

Leur rapport à la terre était le plus éloigné du rapport marchand. Cela faisait d'eux les plus ardents admirateurs de la Camargue sauvage et de la culture qu'elle permet d'exprimer. Ils pouvaient admettre à ce titre un ajustement des pratiques agricoles ou cynégétiques aux besoins de la cause écologiste. Mais ils revendiquaient (ou valorisaient, s'ils étaient là depuis peu) une connaissance intime de " la vraie Camargue ». Selon eux, seul le sud méritait cette appellation en raison de ses milieux

\footnotetext{
25 Un achat envisagé en grande partie pour la chasse perpétue la logique qui prévalait jusqu'en 1830, du temps où la Camargue était aux mains des nobles (Picon, 1978, p. 60).

26 Ce sont donc des rentiers au sens de Walras - associés aux «fermiers » et non étrangers à cette entreprise comme dans la logique de la rente foncière selon Ricardo.
}

naturels plus préservés ainsi que grâce à la présence de grandes manades. Ces propriétaires vantaient les savoirfaire traditionnels des cavaliers ou des chasseurs, estimant que les "écologistes », extérieurs au milieu camarguais, ne sont pas forcément en mesure de faire mieux pour préserver les lieux. Un des descendants des fondateurs du PNRC expliquait : « Mon père a aimé la Camargue et c'est probablement un de ceux qui ont contribué à maintenir une tradition.» Plus que tout autre propriétaire, le rentier estimait que la grande propriété détenue par des gens qui n'ont pas besoin d'exploiter le foncier est la garantie absolue de la préservation du milieu naturel (mais aussi culturel) : «Moi je suis pour la défense des propriétaires riches [...]. La Camargue n'existe que parce qu'il y a eu des Hoffmann, des Pastré, des Fabre $^{27}$. » Pour autant, bien qu'élitistes, ces propriétaires regrettaient rarement l'éviction de la fondation de la gestion du PNRC, dans la mesure où cette dernière était plutôt animée, depuis les années 1980, par des propriétaires défendant des logiques productivistes.

\section{Conclusion}

Au-delà de ces profils, le poids de la grande propriété en Camargue (caractérisée par sa longévité et par la maîtrise ancienne des propriétaires de l'aménagement du territoire) a manifestement fait émerger en ces lieux un sentiment d'appartenance partagé au statut de propriétaire. Mais si la trilogie «travail, liberté, propriété » héritée des idéaux de la Révolution fonctionnait aussi bien comme ferment d'une méfiance vis-à-vis de l'action publique (lorsqu'elle entend imposer ses vues aux personnes privées - a fortiori dans le sens de l'écologisation), il semble que cela était dû précisément à leur dépossession du pouvoir local. Toutefois, bien que le cas camarguais soit quelque peu atypique (rares sont les contextes où la très grande propriété a survécu), cette dimension idéologique des relations entre acteurs publics et propriétaires privés dans la gouvernance environnementale doit être prise en compte. Cette recherche espère avoir mis en relief les implications d'une telle identification : entre désir de reconnaissance et nécessité de participer aux choix et décisions publiques mais aussi, pour les plus idéologues, défense du productivisme.

Face à ce constat - et dans l'attente d'un règlement de la question de la gouvernance locale encore à venir au moment des enquêtes - restait à analyser ce qui permettait, chez certains, une meilleure acceptation des politiques d'écologisation (en réalité, un moindre attrait pour le productivisme). La piste suivie pour ce faire, relative au rapport au foncier des personnes interrogées,

\footnotetext{
27 Sont cités quelques-uns des plus grands propriétaires tant par les superficies possédées que par leur niveau de fortune.
} 
prend tout son sens au regard de la découverte de l'existence d'une catégorie sociale qui se reconnaît avant tout comme propriétaire. Aussi, dans la lignée des travaux consacrés à l'intérêt d'une approche patrimoniale de la terre dans les politiques d'écologisation, il a été recherché une telle conception chez l'un ou l'autre (ou plusieurs) des profils de propriétaires identifiés. L'approche patrimoniale s'est avérée présente pour deux profils, bien que pour des raisons différentes : des facteurs historiques chez les agriculteurs et des facteurs sociologiques chez les rentiers. Pour les premiers, l'enracinement en Provence semble renforcer le lien à l'environnement qui pousse à vouloir préserver la terre ; pour les seconds, en l'absence de besoin de rentrée d'argent, l'attrait pour la Camargue réside dans un attachement au mythe camarguais qui contient l'idée de la préservation d'espaces "sauvages". Se vérifie ici l'affirmation suivante : "Celui qui transmet tient généralement à transmettre, en même tant qu'un bien, des valeurs et des représentations attachées à la propriété » (Godard, 1990, p. 232-233). Ainsi, la valeur identitaire du patrimoine foncier peut contribuer non seulement à minimiser sa valeur économique, mais le hisse également au niveau d'un patrimoine commun géré en conséquence et facilitant l'acceptation d'une gestion patrimoniale publique. Pour affiner encore le propos, dans l'optique d'une pérennisation des stratégies de gestion, respectueuses de l'environnement, il serait intéressant, d'enquêter encore auprès des enfants des propriétaires actuels pour déterminer si la valeur symbolique du patrimoine a une chance de se transmettre aux héritiers.

\section{Références}

Anderson, T.L., Leal, D.R., 2001. Free Market Environmentalism, New York, Palgrave, Revised Edition.

Allard, P., 1978. Fortunes et classes sociales à Arles au XIX siècle. Thèse de $3^{\mathrm{e}}$ cycle, dactylographiée, direction Michel Vovelle, Aix-en-Provence.

Aubertin, C., Vivien, F.-D., 1998. Les Enjeux de la biodiversité, Coll. Poche environnement, Paris, Economica.

Audema, M., 1975. Le Mas de Cabassole : la vie en Camargue entre les deux guerres mondiales, dactylographié (publié en 1986, Les Amis du Vieil Arles, 3).

Barthélémy, C., 2005. Les savoirs locaux : entre connaissances et reconnaissance, VertigO, 6, 1.

Bellivier, F., Noiville, C., 2009. La Bioéquité, Coll. Frontières, Paris, Éditions Autrement.

Billaud, J.-P. (Ed.), 2002. Expérience agri-environnementale française: environnement et gestion des territoires, CNRSMate, Paris, La Documentation française.

Brun, E., Betsch, J.-M., Blandin, P., Humbert, G., Lefeuvre, J.-C., Marinval, M.-C., 2007. Postures des scientifiques et interdisciplinarité dans le champ de l'environnement, Natures Sciences Sociétés, 15, 77-185.
Bromley, D.W., 1991. Environment and Economy: Property Rights and Public Policy, Oxford, Basil Blackwell.

Cadoret, A., 1985. Protection de la nature : histoire et idéologie. De la nature à l'environnement, Coll. Alternatives paysannes, Paris, L'Harmattan.

Capitan, C., 2000. Propriété privée et individu-sujet-de-droits : la genèse historique de la notion de citoyenneté, L'Homme, $153,63-74$.

Castel, R., Haroche, C., 2001. Propriété privée, propriété sociale, propriété de soi: entretiens sur la construction de l'individu moderne, Paris, Fayard.

Claeys-Mekdade, C., 2003. Le Lien politique à l'épreuve de l'environnement: expériences camarguaises, Coll. Ecopolis, Bruxelles, Peter Lang.

Collectif, 1966. Comptes-rendus des Journées nationales d'études sur les parcs naturels régionaux, Lurs-en-Provence, 25 septembre- ${ }^{\text {er }}$ octobre.

Coulomb, P., 1973. Propriété foncière et mode de production capitaliste, Études Rurales, 51, 27-66.

Coulomb, P., 1992. Les étapes du développement agricole français : modèles sociopolitiques et formes de modernisation, Pour, 135, 85-93.

Études rurales, 1988. Numéro spécial «La terre, succession et héritage ", 110, 111, 112.

Godard, O., 1990. Environnement, modes de coordination et systèmes de légitimité : analyse de la catégorie de patrimoine naturel, Revue Économique, 41, 2, 215-241.

Hardin, G., 1968. The tragedy of the commons, Science, 1968, $162,1243-1248$.

Hostiou, R., 1994. La propriété face au droit de l'environnement, Études Foncières, 65, 29-34.

Hermitte, M.A., 1992. La gestion d'un patrimoine commun : l'exemple de la diversité biologique, in Barrère, M. (Ed.), Terre, patrimoine commun, Paris, La Découverte, 120-128.

Hilty, J., Merenlender, A.M., 2003. Studying biodiversity on private lands, Conservation Biology, 17, 132-137.

Isenmann, P., 2003. La Tour du Valat en Camargue: mélanges offerts à Luc Hoffmann en l'honneur de son $80^{e}$ anniversaire, le 23 janvier 2003, Paris, Buchet/Chastel.

Jodelet D., 1984. Représentation sociale : phénomène, concept et théorie, in Moscovici, S., Psychologie sociale, Paris, Puf.

Jollivet, M. (Ed.), 1992. Sciences de la nature, sciences de la société : les passeurs de frontières, Paris, CNRS Éditions.

Malafosse, J., (de), 1977. Un obstacle à la protection de la nature : le droit révolutionnaire, Dix-Huitième Siècle, 9, 91-100.

Marty, P., Vivien, F-D., Lepart, J., Larrère, R. (Coord.), 2005. Les Biodiversités : objets, théories, pratiques, Paris, CNRS Éditions.

Mathevet, R., 2004. Camargue incertaine. Sciences, usages et natures, Paris, Buchet/Chastel.

Mathevet, R., Mauchamp, A., Grillas, P., 2002. Multi-usage et conservation des zones humides ou quel développement durable pour la Camargue ?, Faire Savoirs, 2, 33-39.

Ostrom, E., 1990. Governing the Commons: The Evolution of Institutions for Collective Action, New York, Cambridge University Press.

Ostrom, E., Burger, J., Field, C.B., Norgaard, R.B., Policansky, D., 1999. Revisiting the commons: Local lessons, global challenges, Science, 284, 278-282.

Ost, F., 1995. La Nature hors la loi : l'écologie à l'épreuve du droit, Paris, La Découverte. 
Pailhès, S., 2000. La digue à la mer ou les mésaventures de l'État en Camargue, Provence Historique, 200, 189-205.

Picon, B., Lindenmann, A. (Coord), 2002. Camargues, Faire Savoirs, 2, Sciences humaines et sociales en région Paca.

Picon, B., 1996. Du bon usage de la menace: chronique des représentations de la nature en Camargue, Études Rurales, Éditions EHESS, 141-142, 143-156.

Picon, B., 1978. L'Espace et le Temps en Camargue : essai d'écologie sociale, Arles, Acte Sud.

Pinton, F. (Coord.), 2007. La Construction du réseau Natura 2000 en France, Coll. L'environnement en question, Paris, La Documentation française.

Prevel, M., 2007. L'Usine à la campagne: une ethnographie du productivisme agricole, Paris, L'Harmattan.

Remond-Gouilloud, M., 1989. Du droit de détruire : essai sur le droit de l'environnement, Paris, Puf.

Rifkin, J., 2005. L'Âge de l'accès : la nouvelle culture du capitalisme, Paris, La Découverte.

Savoie, A., Kalahora, B., 1985. La protection des régions de montagne au $\mathrm{XIX}^{\mathrm{e}}$ siècle: forestiers sociaux contre forestiers étatistes, in Cadoret, A. (Ed.), Protection de la nature: histoire et idéologie. De la nature à l'environnement Coll. Alternatives paysannes, Paris, L'Harmattan, 6-23.

Selmi, A., 2006. Administrer la nature: le parc national de la Vanoise, Paris, Éditions de la MSH, Quae.

Reçu le 14 janvier 2010. Accepté le 12 septembre 2012.
Subra de Bieusse, P., 1979. Les Servitudes administratives, Berger-Levrault.

Tamisier, A., 1990. Camargue : milieux et paysages. Évolution de 1942 à 1984, Association pour les recherches en Camargue sur la nature et l'environnement, Arles, Arcane, Sretie, CNRS.

Tamisier, A., Grillas, P., 1994. A review of habitat changes in the Camargue: An assessment of the effects of the loss of biological diversity on the wintering waterfowl community, Biological Conservation, 70, 1, 39-47.

Terry, L.A., Leal, D.R., 2001. Free Market Environmentalism, Palgrave, Macmillan.

Vivien, F.-D., 2001. Le patrimoine naturel : jeux et enjeux entre nature et société, document de travail, in Séminaire Patrimoine, séance du lundi 5 mars 2001, UFR des Sciences économiques et de gestion, Université de Reims Champagne Ardennes.

\section{Documentation du PNRC}

Registres 1970-2000 du conseil d'administration du parc naturel de Camargue

Textes constitutifs du parc naturel régional de Camargue, janvier 1993. 